\title{
An Unusual Presentation of Left Atrial Myxoma in an Adolescent
}

\author{
Deepak Thakura, c, Anuradha Sridhar ${ }^{\mathrm{a}}$, Neville A. G. Solomon ${ }^{\mathrm{b}}$
}

\begin{abstract}
Cardiac myxoma presents an important diagnostic challenge as it may clinically resemble cardiovascular, neurological or systemic diseases. We report an unusual presentation of left atrial myxoma in a 15-yearold girl with features mimicking encephalitis. The tumor was detected by echocardiography incidentally during an episode of hemodynamic instability and an emergency surgical excision was done successfully.
\end{abstract}

Keywords: Myxoma; Cardiac tumors; Echocardiography; Left atrial mass; Benign tumor; Encephalopathy

\section{Introduction}

Myxomas are rare benign primary cardiac tumors in pediatric age group presenting frequently in adult population from third to sixth decade of life. Although benign, they can be life-threatening because they manifest as large space-occupying lesions in the heart. These tumors may cause disruption of the conduction pathways, obstruction of the inflow or outflow tracts and thromboembolic phenomena. If not diagnosed promptly, these benign tumors can be fatal in children.

We present a case of left atrial myxoma in an adolescent with rare presentation as a neurological emergency and indecently diagnosed as a case of left atrial (LA) myxoma by echocardiography done for hemodynamic instability detected in critical care unit. Urgent surgical resection of myxoma was lifesaving with good results. This case emphasizes the importance of non-invasive diagnostic modality like bedside echocardiography as an important evaluation tool for diagnosing primary cardiac tumors like myxoma presenting as a rare cause of life-threatening neurological emergencies in pediatric pa-

Manuscript submitted October 2, 2017, accepted October 25, 2017

aDepartment of Paediatric Cardiology, Apollo Children's Hospital, Chennai, India

bCardio Thoracic Surgery, Apollo Children's Hospital, Chennai, India

'Corresponding Author: Deepak Thakur, Apollo Children's Hospital, 15, Sha-

fee Mohammed Road, Thousand Lights, Chennai, India.

Email: deepak_130505@yahoo.com

doi: https://doi.org/10.14740/ijcp282w tients.

\section{Case Report}

A 15-year-old girl presented with history of high-grade fever of 2 - 3 days duration, and refractory generalized tonic clonic seizures with altered sensorium (status epilepticus) of 3 - 4 h duration prior to presentation in pediatric emergency. The patient had no past history of head trauma, abnormal movements or behavior, headache, visual disturbances, ear discharge, drug intake, insect bite, breathlessness on excretion, palpitations, edema, sore throat, joint pains, skin rashes, bleeding, and menorrhagia. Patient was developmentally normal going good in her school grades, belonging to upper middle class, and resides in urban locality. No significant similar family history was noted. On examination at admission, patient was in altered sensorium with intermittent tonic posturing, no rashes, active bleeding, injury or inset bite marks, pallor, icterus, cyanosis, lymphadenopathy, and edema present. Patient was febrile with temperature of 102 ${ }^{\circ} \mathrm{F}\left(38.9^{\circ} \mathrm{C}\right)$, pulse rate was $110 / \mathrm{min}$, regular, good volume, and blood pressure was $120 / 70 \mathrm{~mm} \mathrm{Hg}$ in right upper limb. Neurological examination showed altered sensorium not responding to commands or painful stimulus (Glasgow coma scale $(\mathrm{GCS})$ score was $<8)$. There was no cranial nerve involvement, tone increased in all limbs, deep tendon reflexes brisk $(3+)$, no neck rigidity, bilaterally normal sized pupils and normal reaction to light. Respiratory system bilateral normal vesicular breath sounds were heard with equal air entry. Cardiovascular system examination S1-S2 heard no added sounds. On abdominal examination, no organomegaly appreciated. With these findings, patient was diagnosed to have encephalitis probably of viral etiology. Seizures were controlled with antiepileptic drugs and in view of low GCS score $(<8)$, patient was intubated and was put on mechanical ventilation in critical care unit. In critical care unit, she had an episode of fluctuating blood pressure measurements with hypotension $(<50$ th percentile for age and height) requiring inotropic support. Lab findings were normal with complete blood count showing $\mathrm{Hb}$ of $11 \mathrm{mg} / \mathrm{dL}$, TLC of 8,500, platelets of $345,000 / \mu \mathrm{L}$ except for erythrocyte sedimentation rate (ESR) which was high $(70 \mathrm{~mm} / \mathrm{h})$, liver function test showed normal bilirubin levels and other parameters, normal renal function test, normal electrolytes and blood sugar level. Viral serology for Japanese encephalitis (JE), PCR for herpes 


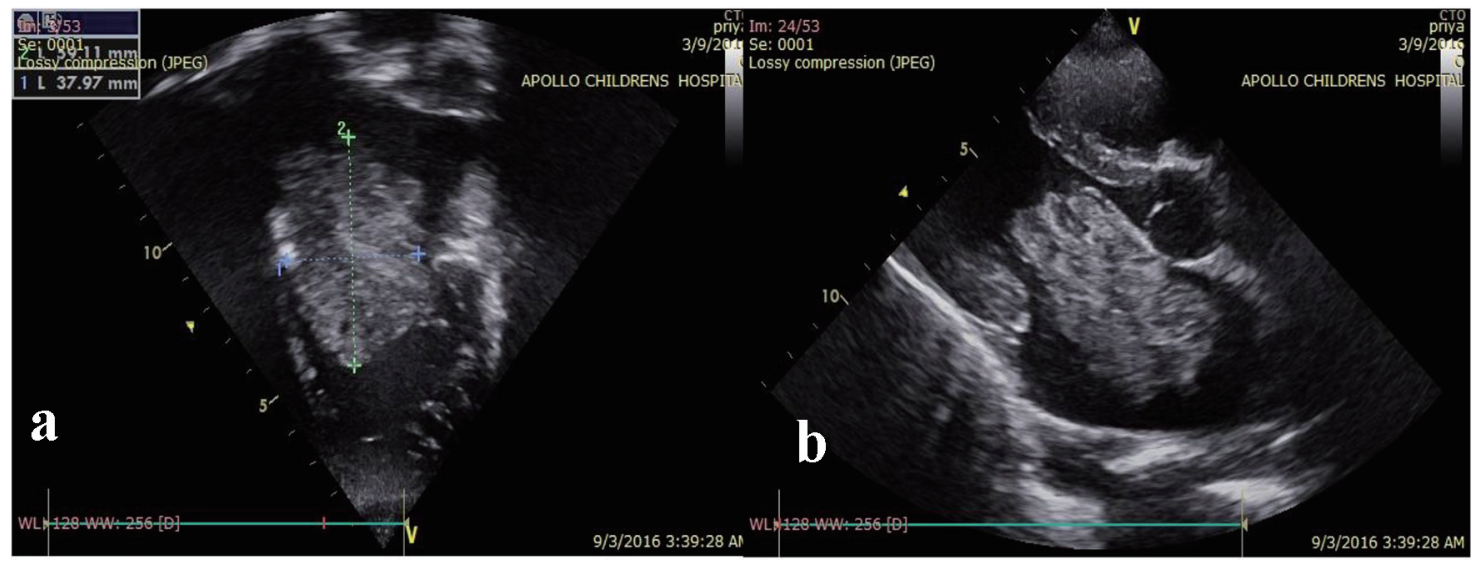

Figure 1. (a, b) Echocardiography showing left atrial mass of homogenous echogenicity, pedunculated, non-cystic, with no calcific focus in four-chamber and parasternal long axis view.

simplex virus (HSV) and blood culture sensitivity were sent. Echocardiography was done to assess ventricular function and rule out cardiac cause for hemodynamic instability. Incidentally echocardiography revealed a large mass $(56 \times 40$ $\mathrm{mm}$ ) in the left atrium attached to lower part of interatrial septum which was of homogenous echogenicity, pedunculated, non-cystic, with no calcific focus (Fig. 1a, b). The mass was impinging on the mitral valve causing severe mitral stenosis (mean inflow gradient of $12 \mathrm{~mm} \mathrm{Hg}$ ) and moderate mitral regurgitation (Figs. 2 and 3). There was severe pulmonary arterial hypertension (estimated right ventricular systolic pressure of $60 \mathrm{~mm} \mathrm{Hg}$ ). Computed tomographic (CT) angiography of brain showed microinfarcts in frontal and parietal cortex. Systemic thromboembolic phenomena due to the left heart myxoma were considered and the patient was started on intravenous heparin infusion. After an informed consent, an emergency surgical excision of mass was done through midline sternotomy on cardiopulmonary bypass through right atrial approach (Fig. 4). Postoperative echocardiography confirmed complete excision of the mass.
There was only mild mitral regurgitation and no evidence of residual mitral inflow obstruction (Fig. 5). Postoperative period was uneventful. Viral markers and blood culture revealed no significant growth. Mechanical ventilation, neuroprotective care and passive physiotherapy were continued for $72 \mathrm{~h}$ postoperatively due to low GCS. Aspirin and low molecular weight heparin were given. She was extubated on the fourth postoperative day when the sensorium improved. Subsequently, she was noticed to have mild left-sided hemiparesis. She was discharged on the seventh postoperative day on aspirin. Patient completely recovered from the neurological deficit at 1 month of postoperative period. Excised mass was gray to red in color, gelatinous, mucoid, soft and friable with irregular margins and hemorrhagic spots suggesting myxoma (Fig. 6). Histopathology examination of the mass showed myxomatous matrix containing stellate and fusiform myxoma cells confirming the diagnosis of atrial myxoma (Fig. 7). During follow-up at 1-year postoperative period, she was neurologically normal and hemodynamically stable with no regrowth of cardiac myxoma. Aspirin was stopped

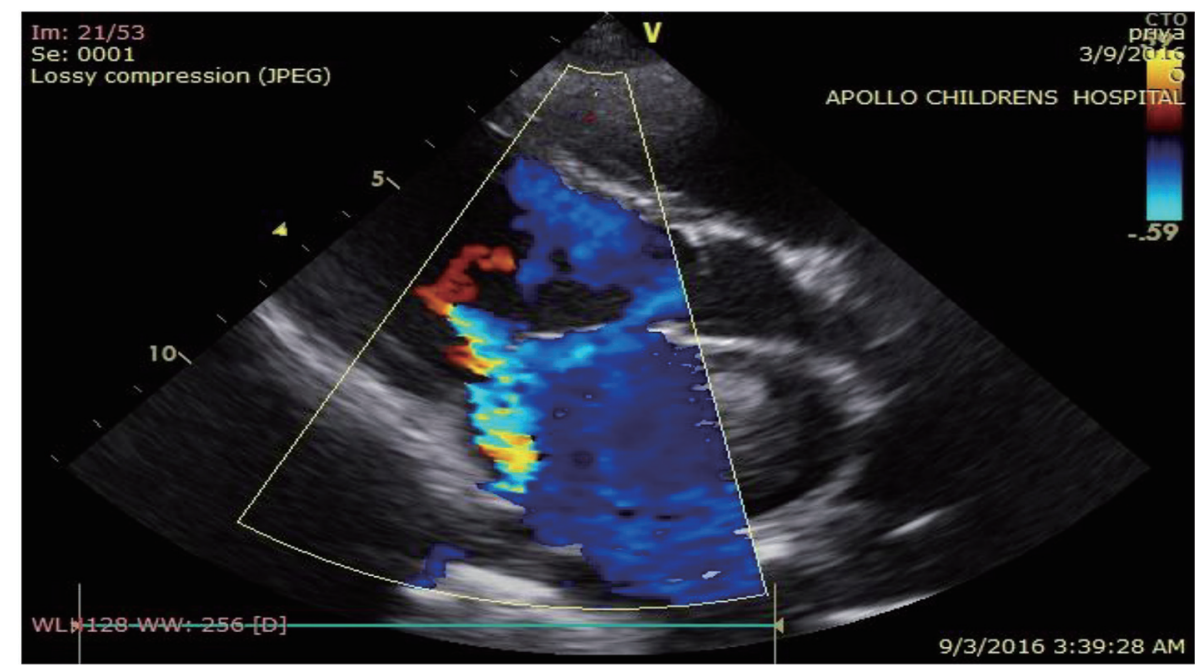

Figure 2. Echocardiography color Doppler showing moderate mitral regurgitation. 


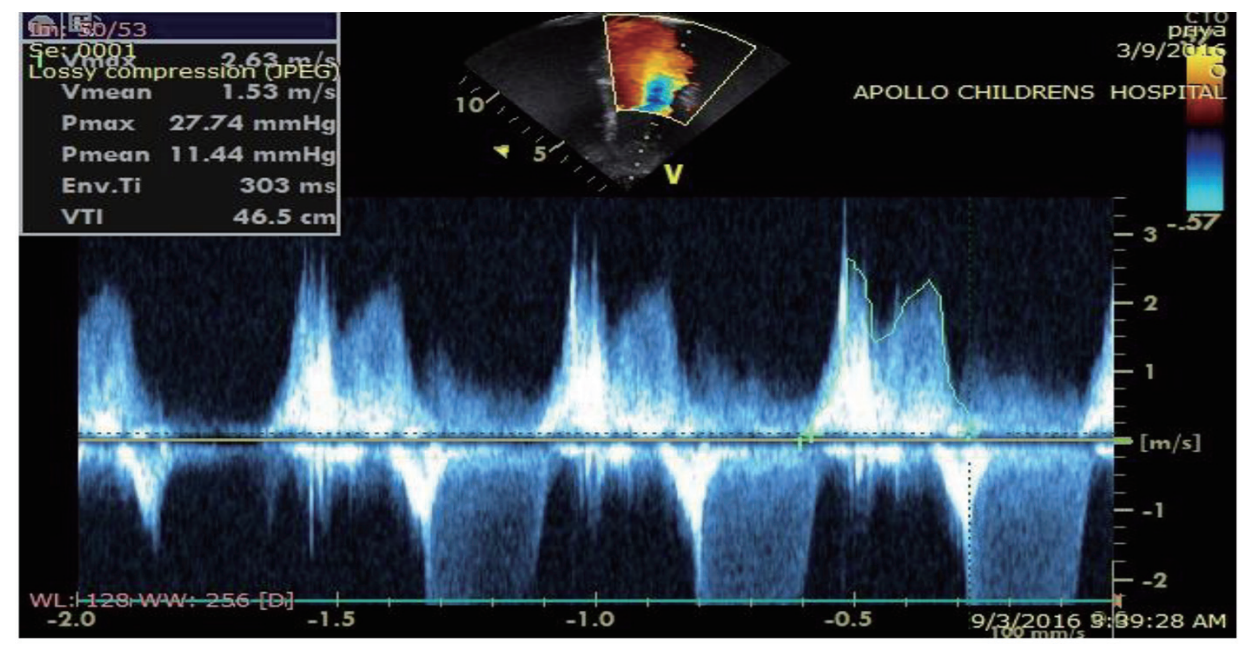

Figure 3. Echocardiography spectral Doppler showing severe mitral stenosis.

and yearly follow-up was advised to monitor for recurrence of myxoma.

\section{Discussion}

Diagnosing an LA myxoma can be difficult because of its rarity and the varied presentation especially in children. The overall incidence of cardiac tumors in pediatric age group is 0.03-0.08\%. Most frequent cardiac tumors are rhabdomyomas (45\%) and fibroids (25\%). Myxoma constitutes only $10 \%$ of all being tumors [1]. This case was presented as a life-threatening neurological emergency (status epilepticus) in an adolescent girl with a short history of fever and sudden onset of seizures. There were no preceding history of significant constitutional, cardiac or neurological symptoms and signs suggesting clinical picture of cardiac myxoma.

Cardiac myxomas presenting as neurological emergency with such a short duration history and normal cardiac findings in adolescent age group are a rare presentation as in most of the studies commonly observed cardiac symptoms and signs

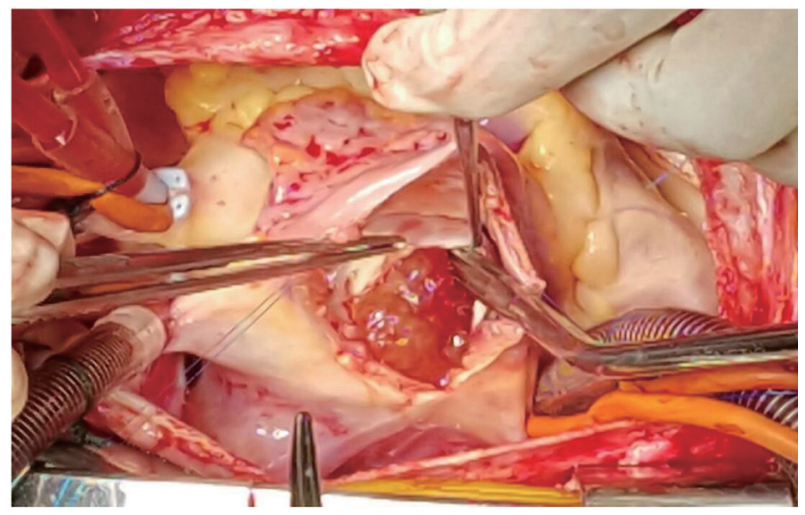

Figure 4. Intraoperative picture of myxoma shown at LA side of inter atrial septum (right atrium opened, interatrial septum opened and held between two forceps). are dyspnea, orthopnea, paroxysmal nocturnal dyspnea, pulmonary edema, cough, hemoptysis, edema, fatigue and mid diastolic murmur or tumor plop is a common cardiac finding [2, 3]. Constitutional symptoms (e.g., fever, weight loss) are seen in around $30 \%$ of patients. Even these constitutional symptoms were not present in our case.

Unlike in our case which presented as neurological emergency on review of various studies in literature patients presented with varied elective neurological findings like dizziness, syncope, hemiparesis, blurred vision, head ache, blindness, and aphasia [4-8]. Atrial tumors may release tumor fragments or thrombi into the systemic circulation, leading to embolization which is usually systemic but can be pulmonic. The most serious complications of such embolization are neurologic [5, 6]. One of the studies shows five of the 11 patients (45\%) with LA myxoma had abnormalities on neurologic examination in the form of hemiparesis and other neurological deficits, and five of five had CT evidence of non-hemorrhagic cerebral infarction [7].

The age of presentation of our case is also rare as myxomas are particularly frequent from the third to the sixth decades of life and show a 2:1 female predominance $[4,5,11]$. There are case reports and case series on pediatric patients presenting with LA myxoma but with wide age of presentation varying from 2 years to 17 years of age [9-16].

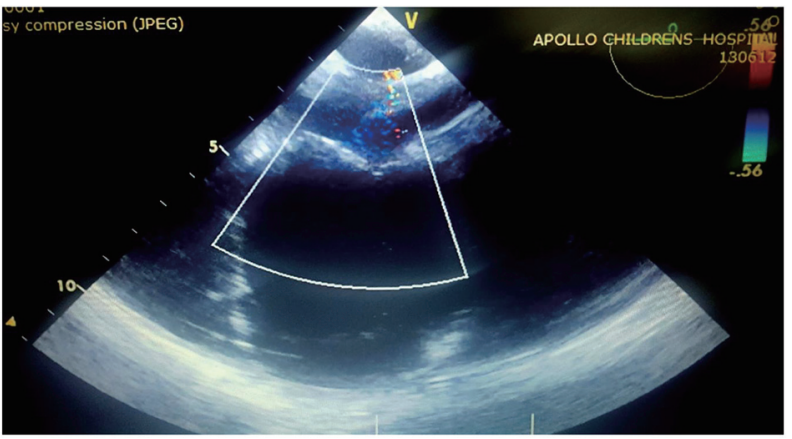

Figure 5. Postoperative echocardiography showing no residual defect causing mitral inflow obstruction. 


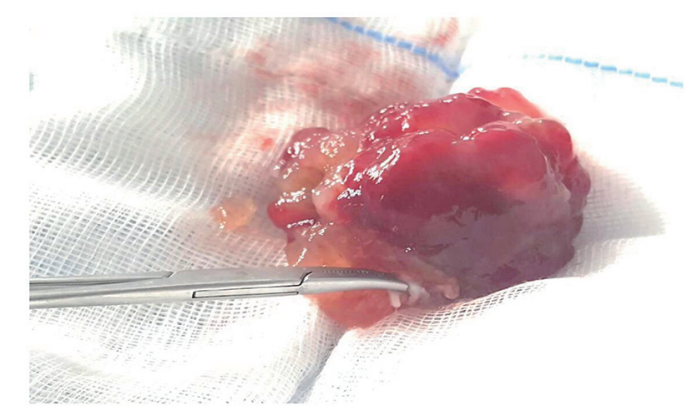

Figure 6. Gross appearance of excised mass showing gelatinous, mucoid, soft and friable with irregular margins and hemorrhagic spots.

Even lab parameters were also unusually normal in our case except for only high ESR value, whereas there are studies where laboratory abnormalities (e.g., anemia and elevations in the ESR, C-reactive protein, or globulin level) were present in $35 \%$ of cardiac myxoma cases with systemic symptoms [3].

Unlike other cases reported in literature as discussed where patients presented for elective evaluation of cardiac, neurological symptoms along with abnormal blood parameters, this case was presented as a neurological emergency with no significant constitutional symptoms, cardiac symptoms, preceding neurological symptoms and findings or significant cardiac findings but for the episodes of fluctuating blood pressure in critical care unit for which urgent echocardiography detected large LA myxoma causing severe mitral valve obstruction and severe pulmonary arterial hypertension (PAH) (indirect evidence of severe mitral valve obstruction). An urgent surgical excision of this myxoma was a lifesaving procedure for this patient as untreated this large LA myxoma would have caused more embolic episodes leading to multiorgan involvement and even worsened neurological condition of the patient. Severe mitral valve obstruction was also a risk factor for low cardiac output and even sudden cardiac arrest too. Overall, the survival of LA myxoma resection has good prognosis with 10-, 20- and 30 -year survival of $77 \%, 52 \%$ and $34 \%$, respectively and operative mortality less than $0.5 \%$ [5].

We conclude that children presenting as a neurological emergency with normal cardiac examination and preceding neurological status should be subjected to cardiac evaluation using echocardiography. This helps in early diagnosis and prompt treatment of potentially life-threatening cardiac conditions like LA myxoma as in this case where due to paucity of cardiac and neurological symptoms and sudden presentation as neurological emergency, LA myxoma was difficult to diagnose on clinical basis.

\section{Funding}

This study was not funded by any agency.

\section{Conflict of Interest}

The authors declare that they have no conflict of interest.

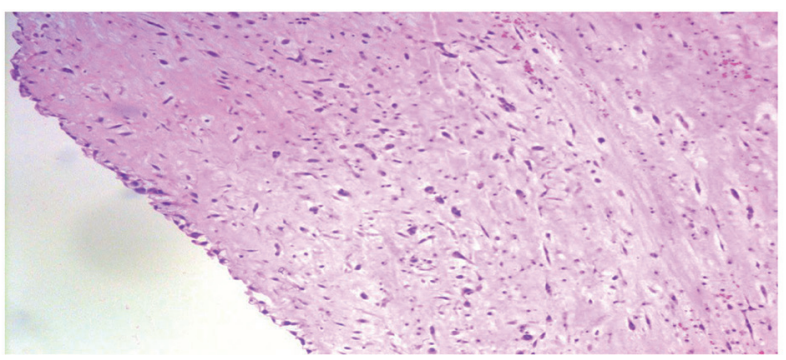

Figure 7. Histopathology showing myxomatous matrix containing stellate and fusiform myxoma cells.

\section{Consent}

Informed consent was obtained from all individual participants included in the study.

\section{References}

1. Becker RP, Frangini SP, Arnaiz GP. [Recurrent left atrial myxoma. Report of a case in a 2 year-old boy]. Rev Med Chil. 2006;134(5):635-640.

2. Kolluru A, Desai D, Cohen GI. The etiology of atrial myxoma tumor plop. J Am Coll Cardiol. 2011;57(21):e371.

3. Aggarwal SK, Barik R, Sarma TC, Iyer VR, Sai V, Mishra $\mathrm{J}$, Voleti CD. Clinical presentation and investigation findings in cardiac myxomas: new insights from the developing world. Am Heart J. 2007;154(6):1102-1107.

4. Goodwin JF. Diagnosis of left atrial myxoma. Lancet. 1963;1(7279):464-468.

5. Pinede L, Duhaut P, Loire R. Clinical presentation of left atrial cardiac myxoma. A series of 112 consecutive cases. Medicine (Baltimore). 2001;80(3):159-172.

6. Burke AP, Gomez-Roman JJ, Loire R. World Health Organization: Tumours of the lung, pleura, thymus and heart. IARC Press; 2004.

7. Knepper LE, Biller J, Adams HP, Jr., Bruno A. Neurologic manifestations of atrial myxoma. A 12-year experience and review. Stroke. 1988;19(11):1435-1440.

8. Shah IK, Dearani JA, Daly RC, Suri RM, Park SJ, Joyce LD, Li Z, et al. Cardiac Myxomas: A 50-year experience with resection and analysis of risk factors for recurrence. Ann Thorac Surg. 2015;100(2):495-500.

9. MacGowan SW, Sidhu P, Aherne T, Luke D, Wood AE, Neligan MC, McGovern E. Atrial myxoma: national incidence, diagnosis and surgical management. Ir J Med Sci. 1993;162(6):223-226.

10. Goldberg HP, Glenn F, Dotter CT, Steinberg I. Myxoma of the left atrium; diagnosis made during life with operative and post-mortem findings. Circulation. 1952;6(5):762-767.

11. Tipton BK, Robertson JT, Robertson JH. Embolism to the central nervous system from cardiac myxoma. Report of two cases. J Neurosurg. 1977;47(6):937-940.

12. Bobo H, Evans OB. Intracranial aneurysms in a child with recurrent atrial myxoma. Pediatr Neurol. 1987;3(4):230232. 
13. Tonz M, Laske A, Carrel T, da Silva V, Real F, Turina M. Convulsions, hemiparesis and central retinal artery occlusion due to left atrial myxoma in child. Eur J Pediatr. 1992;151(9):652-654.

14. Hung PC, Wang HS, Chou ML, Huang SC, Su WJ. Multiple cerebral aneurysms in a child with cardiac myxoma.
J Formos Med Assoc. 1992;91(8):818-821.

15. Bayir H, Morelli PJ, Smith TH, Biancaniello TA. A left atrial myxoma presenting as a cerebrovascular accident. Pediatr Neurol. 1999;21(2):569-572.

16. Landers C, Baumann R, Cottrill CM. Embolic strokes in an 8-year-old girl. Neurology. 2000;55(1):146. 